Amigo Remote Catheter System (Catheter Robotics Inc.) compared with conventional ablation for symptomatic AF, using a catheter capable of measuring myocardial contact force (Smart Touch, Biosense Webster) in conjunction with Carto 3 (Biosense Webster). Left and right wide area circumferential ablation (L/RWACA) was performed in all patients. All RF applications were delivered at $40 \mathrm{~W}$. For both manual and robotic procedures, the operator and assisting staff were blinded to the force data. This data was instead analysed retrospectively by another physician who was blinded to the technique used. In each case, force data was collected on a point-by-point basis, dividing the WACA lesions into regions using a clock model, with lower numbers being represented anteriorly, higher numbers posteriorly (see figure 1 ) The defined regions were: superior and inferior positions (12 and 6 o'clock), between LUPV and LAA (1 o'clock)/anterior RUPV (1-2 o'clock), anterior L/RLPV (4-5), posterior L/RUPV (7-8), and anterior/posterior to the carina (3/6). Statistical analysis was performed using $R$, applying planned contrasts within a mixed-model design ANOVA to appropriately transformed data.

Results Force data was collected and regionalised from 10 robotic and 8 manual cases. A total of 3329 ablation points were analysed, acquiring 662471 pieces of force data. On the LWACA, the overall mean force applied from the Amigo was $8.2 \mathrm{~g}$, compared with $7.3 \mathrm{~g}$ manually, whilst for the RWACA, the Amigo applied a mean force of $10.2 \mathrm{~g}$, compared with $9.3 \mathrm{~g}$ manually $(\mathrm{p}<0.05$ for all comparisons). On a regional basis, only the anterior RLPV showed a significantly increased mean force using the robot compared with a manual technique (see figure 2). Higher than average force values were observed in anterior RUPV region using both techniques.

Discussion Higher contact force has been associated with increased lesion size and depth, as well as lower rates of acute pulmonary vein reconnection and AF recurrence. However, elevated force is also correlated with increased thrombus formation and steam generation, which are important contributory factors to the generation of cerebral microemboli. In addition, the risk of RF energy delivery varies depending on the location within the left atrium.

074

\section{HUMAN VS ROBOT: A COMPARISON OF CATHETER CONTACT FORCE APPLICATION FOR AF ABLATION (A MAST-AF SUBSTUDY)}

G S Chu, ${ }^{1}$ N Masca, ${ }^{2}$ T P Almeida, ${ }^{3}$ P D Brown ${ }^{4}$ F S Schlindwein, ${ }^{3}$ G A Ng ${ }^{5}$ ${ }^{1}$ University of Leicester, Department of Cardiovascular Sciences; ${ }^{2}$ Cardiovascular Biomedical Research Unit, University of Leicester; ${ }^{3}$ Department of Bioengineering, University of Leicester; ${ }^{4}$ Department of Cardiovascular Sciences, University of Leicester; ${ }^{5}$ Leicester NIHR Biomedical Research Unit in Cardiovascular Disease

\section{doi:10.1136/heartjnl-2013-304019.74}

Introduction It is not known whether there is any difference in catheter force application and therefore effective lesion creation between robotic and human procedures. This may impact on procedural efficacy and clinical outcome.

Method MAST-AF (Manual vs Amigo SmartTouch AF Study) is an ongoing prospective randomised trial (NCT01583855) of the

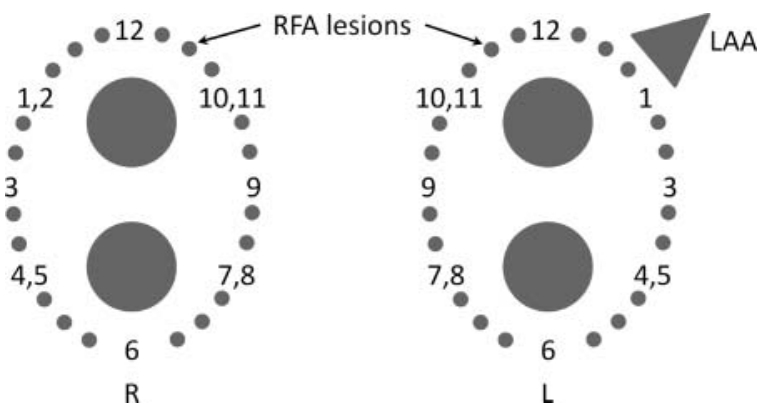

Figure 1

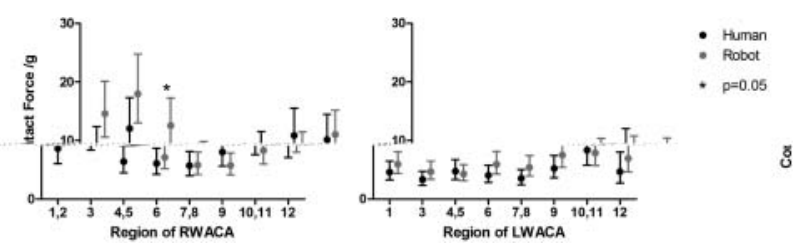

Figure 2 
Consequently, an awareness of catheter force is potentially crucial in ensuring safe yet efficacious AF ablation.

The rather low values we have recorded overall suggest operator overestimation of force application: nevertheless PVI was demonstrated in all patients post-ablation. Higher force around the aorta has been noted previously, and our results are consistent with this (RWACA regions 1-3).

Overall, our initial findings suggest a difference in the performance of robot and human AF ablation. The clinical impact of our findings will be evaluated as part of the scheduled 1 year follow-up for the MAST-AF trial. 